\title{
Malignant psoas syndrome associated with gynecological malignancy: Three case reports and a review of the literature
}

\author{
SHIRO TAKAMATSU ${ }^{1}$, KOSUKE MURAKAMI $^{1}$, HISAMITSU TAKAYA ${ }^{1}$, TAKAKO TOBIUME ${ }^{1}$, \\ HIDEKATSU NAKAI $^{1}$, AYAKO SUZUKI ${ }^{1}$, MASAKI MANDAI ${ }^{2}$ and NORIOMI MATSUMURA ${ }^{1}$ \\ ${ }^{1}$ Department of Obstetrics and Gynecology, Kindai University Faculty of Medicine, Osakasayama, Osaka 589-8511; \\ ${ }^{2}$ Department of Gynecology and Obstetrics, Kyoto University Graduate School of Medicine, Kyoto, Osaka 606-8507, Japan
}

Received February 23, 2018; Accepted May 21, 2018

DOI: $10.3892 / \mathrm{mco} .2018 .1635$

\begin{abstract}
Malignant psoas syndrome (MPS) is a rare and unique cancer-associated syndrome caused by the malignant involvement of the psoas major muscle, and is characterized by ipsilateral lumbosacral plexopathy and painful hip flexion. The pain in MPS is often distressing and intractable, and there is no established effective treatment approach. Herein, the present study reports on three cases of MPS associated with gynecological malignancies, wherein symptom improvement was observed following chemotherapy or radiotherapy. Among 39 cases documented in the literature, female genital tract malignancies were the most frequent causes of MPS; however, the condition may be under-diagnosed, owing to the lack of general recognition. Considering the development of recent high-precision radiation therapy, palliative radiotherapy may serve an important role in the management of MPS. For physicians treating gynecological cancers, early detection of MPS is clinically important as this may allow patients to receive possible therapies and improve their quality of life in end-stage cancer. Further prospective studies should be performed to evaluate effective therapeutic approaches for MPS.
\end{abstract}

\section{Introduction}

Malignant psoas syndrome (MPS) was first described by Stevens and Gonet in 1990 and is a unique cancer-related syndrome characterized by ipsilateral proximal lumbosacral

Correspondence to: Professor Noriomi Matsumura, Department of Obstetrics and Gynecology, Kindai University Faculty of Medicine, 377-2 Ohnohigashi, Osakasayama, Osaka 589-8511, Japan

E-mail: noriomi@med.kindai.ac.jp

Abbreviations: CT, computed tomography; EBRT, external beam radiation therapy; IMRT, intensity modulated radiation therapy; MPS, malignant psoas syndrome; TC, paclitaxel plus carboplatin

Key words: malignant psoas syndrome, gynecological cancer, radiotherapy, lumbosacral plexopathy, palliative radiotherapy plexopathy and painful hip flexion caused by radiographically or pathologically evident malignant involvement of the psoas major muscle $(1,2)$. MPS is a rare entity, and its incidence has been reported to be less than $1 \%$ among patients with high-risk cancer, although this value may reflect under-recognition of the disease $(3,4)$.

As the pain in MPS involves a combination of nociceptive and neuropathic pain, it is often intractable and refractory to multiple drug treatment. In addition, MPS usually occurs in patients with advanced and recurrent cancer; thus, it is difficult to control the symptoms, resulting in remarkable deterioration in the quality of life of patients. Although several studies have reported different treatment approaches for MPS, the effectiveness appears to be limited and an evidence-based effective protocol remains to be established.

In the present paper, we report three cases of MPS associated with gynecological malignancies, which showed symptom improvement with particular medical interventions. We also discuss the clinical features and practical treatment of MPS with a review of the literature.

\section{Case reports}

Written and verbal informed consent was obtained from all participating individuals.

Case 1. A 31-year-old woman was diagnosed with stage IB poorly differentiated squamous cervical cancer and was treated with radical hysterectomy and bilateral salpingo-oophorectomy followed by adjuvant chemotherapy with paclitaxel plus carboplatin (TC). Six months after the completion of the initial treatment, a recurrent tumor was detected in the lower abdominal wall on follow-up computed tomography (CT) scan. Abdominal wall resection and pedicle flap reconstruction using the right tensor fascia lata were performed. However, 3 months after the surgery, pelvic lymph node metastases were identified. External beam radiation therapy (EBRT) to the whole pelvis (46 Gy in 23 fractions) and bilateral inguinal region (20 Gy in 10 fractions) was performed with a total dose of $66 \mathrm{~Gy}$ in 33 fractions (Fig. 1A) and partial tumor regression was observed. Although adjuvant chemotherapy was considered, the patient was hospitalized again because of small bowel obstruction. She eventually discontinued the additional therapy. 
Thirty months after the last radiation therapy, she presented with a complaint of severe low back pain. The pain was left-sided, extending from the lower back through the hip and thigh to inside the knee, and it worsened in the last 1 month. Physical examination identified painful flexion of her left hip and gait abnormality.

The patient had been administered tramadol $200 \mathrm{mg} /$ day and loxoprofen $180 \mathrm{mg} /$ day for pain control in outpatient settings, although this did not work well. After hospital admission, the medications were changed to oral long-acting oxycodone $20 \mathrm{mg} /$ day plus a rescue dose of short-acting oxycodone $5 \mathrm{mg}$, naproxen $300 \mathrm{mg} /$ day, acetaminophen $4 \mathrm{~g} / \mathrm{day}$, and pregabalin $75 \mathrm{mg} /$ day. Subsequently, betamethasone $4 \mathrm{mg}$ /day was added, and the dose of oxycodone was increased to $60 \mathrm{mg} / \mathrm{day}$. However, her pain remained uncontrollable and was associated with an unpleasant sleepiness. Magnetic resonance imaging revealed bilateral metastatic para-aortic lymphadenopathy (Fig. 2). The left nodes were apparently invading laterally into the left psoas muscle and almost replacing it. Although the culprit lesion was very close to the previous radiation field, intensity modulated radiation therapy (IMRT) targeting the area was proposed by the radiologist (Fig. 1B-D).

After IMRT, with a total dose of 45 Gy in 25 fractions, her pain gradually reduced. Finally, she recovered normal gait and was discharged from the hospital to a local clinic for terminal care. Her pain relapsed 4 months after discharge but was well controlled with administration of opioids. The patient died due to the disease 8 months after symptom onset of MPS.

Case 2. A 63-year-old woman underwent initial surgery involving total hysterectomy, bilateral salpingo-oophorectomy, and pelvic lymph adenectomy for stage IB uterine endometrial serous adenocarcinoma, followed by adjuvant chemotherapy with TC. Although follow-up studies for the next 4 years showed no evidence of recurrent disease, CT detected multiple para-aortic lymph node metastases. The nodes were sensitive to salvage chemotherapy with TC and were resected by the following surgery. However, soon after the completion of the additional chemotherapy with the same regimen, multiple metastatic lesions in bilateral lung fields and supraclavicular lymph nodes were observed. Subsequently, she received intermittent chemotherapy with a regimen of docetaxel and carboplatin; however, there was limited tumor response.

After 5 cycles of the chemotherapy, she gradually complained of worsening muscle weakness, edema, and severe pain with a burning sensation in the right leg. On hospital admission for treatment, her right hip was fixed in the flex position with walking difficulty.

Analgesics including oral long-acting oxycodone $10 \mathrm{mg} / \mathrm{day}$, loxoprofen $180 \mathrm{mg} / \mathrm{day}$, and acetaminophen $3 \mathrm{~g} /$ day were initially administered. The dose of oxycodone was increased to $40 \mathrm{mg} /$ day, and a variety of adjuvant drugs were administered, such as pregabalin $50 \mathrm{mg} /$ day, olanzapine $2.5 \mathrm{mg}$ at night, and betamethasone $4 \mathrm{mg} /$ day. However, her condition did not show significant improvement. Subsequent CT revealed para-aortic lymph node metastases invading into the right psoas muscle (Fig. 3). She refused chemotherapy because of the possible adverse effects and was referred to radiologists for palliative radiotherapy. EBRT to the culprit lesion was planned, with a dose of 40 Gy in 16 fractions (Fig. 4). Two weeks after the initiation of radiation, her pain significantly improved, and analgesics were stopped. Subsequently, she could walk independently and was discharged from the hospital to home. The symptoms of MPS did not recur; however, she died due to the disease, 7 months after symptom onset.

Case 3. A 50-year-old woman with an initial diagnosis of stage IVB fallopian tube cancer was referred to our hospital for treatment. She presented with abdominal distension and severe pain radiating from her left lower back to the lower leg. The pain was associated with an inability to extend the left hip, and thus, the patient was forced to rest with the hip in flexion.

$\mathrm{CT}$ revealed cancerous ascites, multiple abdominal disseminations, systematic lymphadenopathy, and multiple intramuscular space-occupying lesions at the left psoas major and quadratus lumborum muscles (Fig. 5). Pain control medication was initiated, including oral long-acting oxycodone $20 \mathrm{mg} / \mathrm{day}$, naproxen $300 \mathrm{mg} / \mathrm{day}$, and acetaminophen $4 \mathrm{~g} / \mathrm{day}$, with a rescue dose of short-acting oxycodone $5 \mathrm{mg}$. However, the required dose of oxycodone continuously increased every day, and opioid-induced side effects, including nausea, vomiting, drowsiness, and delirium, progressively became intolerable. Opioid switching to intravenous fentanyl was initiated, but it was not effective. The pain worsened and became agonizing. After diagnostic laparotomy, the patient was enrolled in a phase 3 clinical trial with conventional TC chemotherapy plus an investigational drug or placebo.

As the treatment advanced, her symptoms improved. Finally, when the 6-course treatment was completed, radiographic complete tumor remission was achieved, and her symptoms almost disappeared.

At 7 months after the completion of chemotherapy, recurrence was observed at the para-aortic lymph nodes and intra psoas major muscle with no subjective symptoms. Salvage chemotherapy with TC plus bevacizumab was undertaken, and the recurrent tumors disappeared. The patient is currently alive with no evidence of the disease.

\section{Literature review and discussion}

MPS is considered to be a rare entity. The frequency of lumbosacral plexopathy in patients with cancer, which is not specific to MPS, has been reported as $0.71 \%$ (4). However, such a low frequency may reflect significant under-recognition of MPS $(3,4)$. In fact, we encountered our three patients over a period of 1 year in our hospital. Similar cases may have been previously overlooked.

On reviewing the English literature, we identified 39 cases of MPS in which the primary cancer site was identified (1-3,5-19) (Table I). It should be noted that among the different primary cancers, female genital tract malignancies, accounting for $27 \%$ (10/39) of the cases, were the most common diseases causing MPS, followed by gastrointestinal $(20 \%, 8 / 39)$ and urinary tract $(20 \%, 8 / 39)$ malignancies. Psoas muscle involvement in MPS is considered to result from either direct intramuscular metastasis or extra-nodal extension from regional lymph nodes $(2,3)$. Considering this mechanism, it is reasonable that cancers with a propensity to lymphatically metastasize to the retroperitoneal nodes and/or hematogenously to the psoas 
A

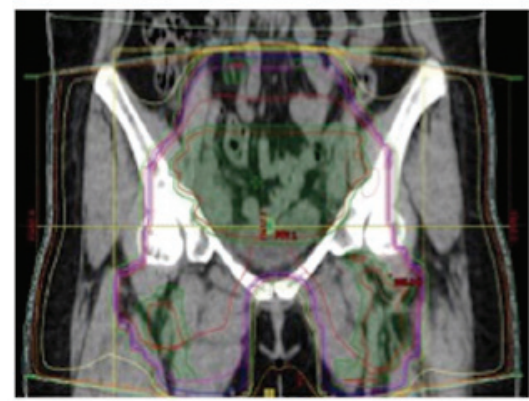

C

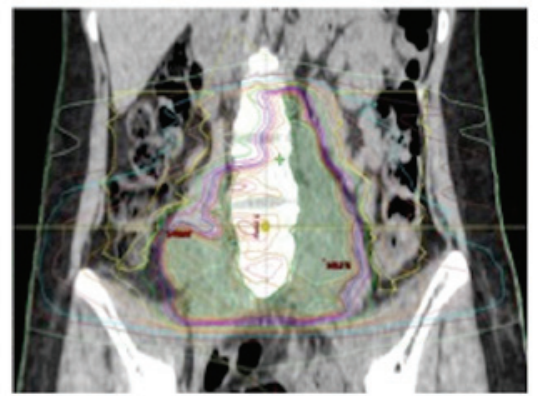

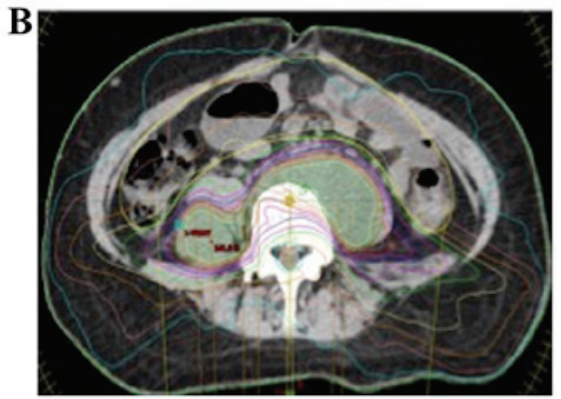

D

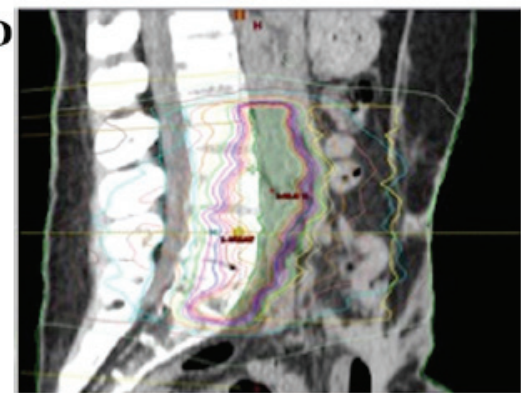

Figure 1. Dose distribution of the initial and second radiation therapy. (A) Coronal plane view of external beam radiation therapy. (B) Axial, (C) coronal and (D) sagittal plane views of intensity modulated radiation therapy.

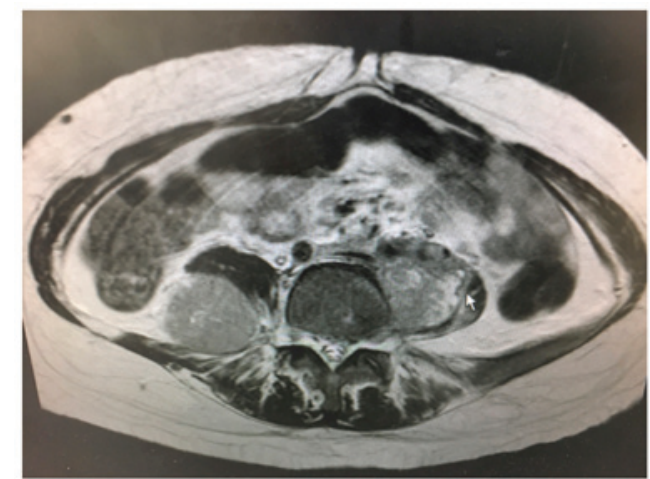

Figure 2. T2-weighted magnetic resonance imaging in the axial plane. Bilateral para-aortic lymph nodal metastases are observed. The left nodes infiltrate into and almost replace the psoas muscle.

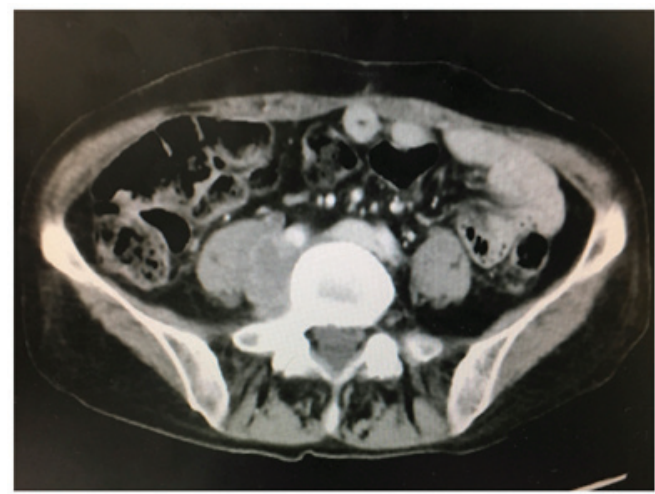

Figure 3. Abdominal contrast-enhanced computed tomography in the axial plane. Right para-aortic nodal metastasis is observed. The node invades from the lateral surface of the vertebral body into the right psoas muscle.

major muscle, such as that observed in genitourinary and colorectal malignancies, are likely to cause MPS.

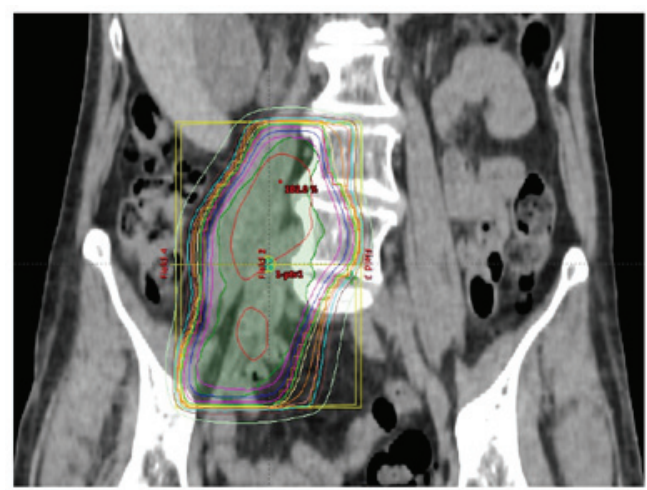

Figure 4. Sagittal plane view of dose distribution of external beam radiation therapy.

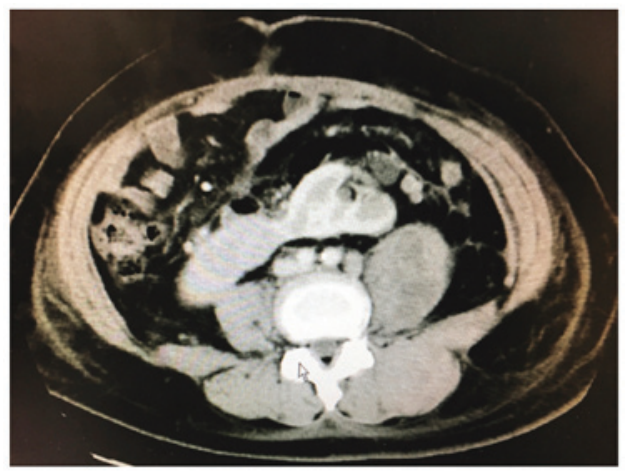

Figure 5. Abdominal contrast-enhanced computed tomography in the axial plane. Multiple metastases are observed in the bilateral para-aortic lymph nodes, left psoas major muscle and left quadratus lumborum muscle. The patient shows a congenital horseshoe kidney.

The pain associated with MPS is usually difficult to treat. This could be explained by the complex pathogenesis of this 
Table I. Sites of primary cancer causing malignant psoas syndrome reported in the literature.

\begin{tabular}{|c|c|c|c|}
\hline Authors, year & $\begin{array}{l}\text { Site of primary cancer } \\
\text { female genital tract }\end{array}$ & Number of cases (n) & (Refs.) \\
\hline $\begin{array}{l}\text { Agar et al, 2004; Stevens et al, 2010; } \\
\text { Takase et al, 2015; Basu et al, 2014; } \\
\text { Ampil et al, 2001; Bar-Dayan et al, 1997; } \\
\text { Yamaguchi et al, } 2017\end{array}$ & Uterine cervix & 7 & $(2,3,6,8,11,14,19)$ \\
\hline Agar et al, 2004 & Ovary & 2 & (2) \\
\hline Yang et al, 1999 & $\begin{array}{l}\text { Uterine endometrium } \\
\text { Gastrointestinal tract }\end{array}$ & 1 & (10) \\
\hline $\begin{array}{l}\text { Yang et al, 1999; Ampil et al, 2001; } \\
\text { Avery et al, } 1988\end{array}$ & Colon & 5 & $(10,11,13)$ \\
\hline Yang et al, 1999 & Rectum & 1 & $(10)$ \\
\hline Ota et al, 2017 & Stomach & 1 & (9) \\
\hline Ampil et al, 2001 & $\begin{array}{l}\text { Pancreas } \\
\text { Urinary tract }\end{array}$ & 1 & (11) \\
\hline Stevens et al, 1990; Yamaguchi et al, 2017 & Bladder & 3 & $(1,19)$ \\
\hline Stevens et al, 1990; Takase et al, 2015 & Prostate & 2 & $(1,6)$ \\
\hline Ampil et al, 2001; Linn et al, 1996 & Kidney & 2 & $(11,15)$ \\
\hline Takase et al, 2015 & $\begin{array}{l}\text { Urachus } \\
\text { Hematological cancer }\end{array}$ & 1 & (6) \\
\hline Agar et al, 2004; Kounami et al, 2012 & $\begin{array}{l}\text { Lymphoma Lymphoma } \\
\text { Others }\end{array}$ & 4 & $(2,7,12,17)$ \\
\hline Ampil et al, 2001; Nash et al, 1996 & Lung & 2 & $(11,18)$ \\
\hline Elkowitz et al, 1991 & Tongue & 1 & (16) \\
\hline Elkowitz et al, 1991 & Nasopharynx & 1 & (16) \\
\hline Ampil et al, 2001; Yamaguchi et al, 2017 & Skin & 2 & $(11,19)$ \\
\hline McKay et al, 2017 & Psoas muscle & 1 & (5) \\
\hline
\end{tabular}

condition, with malignant involvement of the psoas major muscle producing not only deep somatic nociceptive pain but also neuropathic pain, owing to lumbosacral plexopathy $(2,3)$. Furthermore, patients presenting with MPS usually have advanced and recurrent cancers. In the case of initially diagnosed cancer, cancer-directed therapy can be the most effective option; however, in most cases, the tumor is already refractory to standard therapy. Consequently, only palliative treatments could be adapted in such cases. Among our cases, the patient with primary fallopian tube cancer showed symptom improvement concordant with the chemotherapeutic effect.

Several reports have proposed approaches for the management of MPS, but no single, crucial protocol has been established. A simple analgesic agent is often ineffective, and multimodal pharmacotherapy, including opioids, non-opioid analgesics, and adjuvant drugs (antiepileptics, antidepressants, and muscle relaxants), is required, although its effectiveness has been shown to be limited $(2,3)$. Recent studies have shown that rotation switching of opioids to methadone was effective for refractory neuropathic pain $(6,20)$. Other studies reported the efficacy of intervention strategies, including epidural analgesia $(9,19)$, injection from a psoas sheath catheter $(21)$ or intrathecal catheter $(19,22)$, and dorsal rhizotomy (23). Radiotherapy is occasionally adapted for both cancer-directed and palliative therapy, although its efficacy remains to be clarified $(1,2,4,5)$. In two of our cases with progressive recurrent uterine cancer, palliative radiation therapy resulted in significant symptom improvement. It should be noted that in the cervical cancer case, IMRT targeted to the lesion close to the post-radiated area was effective without serious adverse events. In recent decades, high-precision radiation therapy, including IMRT, has been developed. As this therapy can deliver a highly conformal dose to the target with minimal exposure to adjacent normal tissues, it can significantly reduce toxicity and improve disease control $(24,25)$. It could be emphasized that radiotherapy is a considerable treatment option for MPS.

The survival of patients with MPS is considered to be very short, as demonstrated by many previous studies, and the reported median survival duration was 5.5-10.7 months after diagnosis $(2,4,19,23)$. In the present study, patients in cases 1 and 2 died at 8 and 7 months from the time of diagnosis, respectively. Considering such a significant mortality rate and 
remarkable impact on the patient's quality of life, the recognition and diagnosis of this syndrome are clinically important to allow patients to receive all possible therapies.

\section{Acknowledgements}

Not applicable.

\section{Funding}

No funding was received.

\section{Availability of data and materials}

All data generated or analyzed during this study are included in this published article.

\section{Authors' contributions}

ST designed the study and prepared the manuscript draft and substantively participated in revising the manuscript. NM contributed in analyzing the patients' data and revised the manuscript. KM, HT, TT, and HN collected and interpreted the clinical data and conducted a comprehensive literature review related to this project. AS and MM helped revise the manuscript for important intellectual content. All authors thoroughly reviewed the manuscript. All authors read and approved the final version of this manuscript.

\section{Ethics approval and consent to participate}

The present study was conducted in accordance with the ethical principles of the Declaration of Helsinki. Written and verbal informed consent was obtained from all participating individuals.

\section{Consent for publication}

Not applicable.

\section{Competing interests}

The authors declare that they have no competing interests.

\section{References}

1. Stevens MJ and Gonet YM: Malignant psoas syndrome: Recognition of an oncologic entity. Australas Radiol 34: 150-154, 1990.

2. Agar M, Broadbent A and Chye R: The management of malignant psoas syndrome: Case reports and literature review. J Pain Symptom Manage 28: 282-293, 2004.

3. Stevens MJ, Atkinson C and Broadbent AM: The malignant psoas syndrome revisited: Case report, mechanisms, and current therapeutic options. J Palliat Med 13: 211-216, 2010.

4. Jaeckle KA: Neurological manifestations of neoplastic and radiation-induced plexopathies. Semin Neurol 24: 385-393, 2004.

5. McKay TA, Bishop S and McKay MJ: Primary psoas sarcoma causing malignant psoas syndrome: Favourable response to radiotherapy. Ann Transl Med 5: 105, 2017.
6. Takase N, Ikegaki J, Nishimura H, Yuasa S, Ito Y and Kizawa Y: Methadone for patients with malignant psoas syndrome: Case series of three patients. J Palliat Med 18: 645-652, 2015.

7. Kounami S, Shibuta K, Yoshiyama M, Mitani Y, Watanabe T, Takifuji K and Yoshikawa N: Primary anaplastic large cell lymphoma of the psoas muscle: A case report and literature review. Acta Haematol 127: 186-188, 2012.

8. Basu S and Mahajan A: Psoas muscle metastasis from cervical carcinoma: Correlation and comparison of diagnostic features on FDG-PET/CT and diffusion-weighted MRI. World J Radiol 6: 125-129, 2014.

9. Ota T, Makihara M, Tsukuda H, Kajikawa R, Inamori M, Miyatake N, Tanaka N, Tokunaga M, Hasegawa Y, Tada T and Fukuoka M: Pain management of malignant psoas syndrome under epidural analgesia during palliative radiotherapy. J Pain Palliat Care Pharmacother 31: 154-157, 2017.

10. Yang WT, Yeo W and Metreweli C: Imaging of iliopsoas metastasis. Clin Radiol 54: 85-89, 1999.

11. Ampil FL, Lall C and Datta R: Palliative management of metastatic tumors involving the psoas muscle: Case reports and review of the literature. Am J Clin Oncol 24: 313-314, 2001.

12. Connor SE, Chavda SV and West R: Recurrence of non-Hodgkin's lymphoma isolated to the right masticator and left psoas muscles. Eur Radiol 10: 841-843, 2000.

13. Avery GR: Metastatic adenocarcinoma masquerading as a psoas abscess. Clin Radiol 39: 319-320, 1988.

14. Bar-Dayan Y, Fishman A, Levi Z and Rachmani R: Squamous cell carcinoma of the cervix with psoas abscess-like metastasis in an HIV-negative patient. Isr J Med Sci 33: 674-676, 1997.

15. Linn JF, Fichtner J, Voges G, Schweden F, Störkel S and Hohenfellner R: Solitary contralateral psoas metastasis 14 years after radical nephrectomy for organ confined renal cell carcinoma. J Urol 156: 173, 1996.

16. Elkowitz SS, Patel M and Hirschfield LS: Paravertebral muscle metastases from primary tongue and nasopharyngeal carcinomas. Clin Radiol 43: 400-401, 1991.

17. Cortés-Blanco A and Martínez-Lázaro R: Lymphoma mimicking a psoas abscess on Ga-67 scintigraphy. Clin Nucl Med 25: $567-568,2000$.

18. Nash S and Rubenstein J, Chaiton A and Morava-Protzner I: Adenocarcinoma of the lung metastatic to the psoas muscle. Skeletal Radiol 25: 585-587, 1996.

19. Yamaguchi T, Katayama K, Matsumoto M, Sato Y, Nakayama N and Hisahara K: Successful control of pain from malignant psoas syndrome by spinal opioid with local anesthetic agents. Pain Pract: 23 Sep 2017 (Epub ahead of print).

20. Porta-Sales J, Garzón-Rodríguez C, Villavicencio-Chávez C, Llorens-Torromé S and González-Barboteo J: Efficacy and safety of methadone as a second-line opioid for cancer pain in an outpatient clinic: A prospective open-label study. Oncologist 21: 981-987, 2016.

21. Douglas I and Bush D: The use of patient-controlled boluses of local anaesthetic via a psoas sheath catheter in the management of malignant pain. Pain 82: 105-107, 1999.

22. Becker R, Jakob D, Uhle EI, Riegel T and Bertalanffy H: The significance of intrathecal opioid therapy for the treatment of neuropathic cancer pain conditions. Stereotact Funct Neurosurg 75: 16-26, 2000.

23. Son BC, Yoon JH, Kim DR and Lee SW: Dorsal rhizotomy for pain from neoplastic lumbosacral plexopathy in advanced pelvic cancer. Stereotact Funct Neurosurg 92: 109-116, 2014.

24. Nakamura K, Sasaki T, Ohga S, Yoshitake T, Terashima K, Asai K, Matsumoto K, Shioyama Y and Honda H: Recent advances in radiation oncology: Intensity-modulated radiotherapy, a clinical perspective. Int J Clin Oncol 19: 564-569, 2014.

25. Zhang MX, Li J, Shen GP, Zou X, Xu JJ, Jiang R, You R, Hua YJ, Sun Y, Ma J, et al: Intensity-modulated radiotherapy prolongs the survival of patients with nasopharyngeal carcinoma compared with conventional two-dimensional radiotherapy: A 10-year experience with a large cohort and long follow-up. Eur J Cancer 51: 2587-2595, 2015. 